\section{( OPEN ACCESS}

\title{
Lamivudine/telbivudine-associated neuromyopathy: neurogenic damage, mitochondrial dysfunction and mitochondrial DNA depletion
}

\author{
Hongliang $\mathrm{Xu},{ }^{1}$ Zhaoxia Wang, ${ }^{1}$ Lemin Zheng, ${ }^{2}$ Wei Zhang, ${ }^{1} \mathrm{He} \mathrm{Lv}{ }^{1}$ \\ Suqin Jin, ${ }^{1}$ Yun Yuan ${ }^{1}$
}

- Additional material is published online. To view please visit the journal (http:// dx.doi.org/10.1136/jclinpath2013-202069)

${ }^{1}$ Department of Neurology, Peking University First Hospital, Beijing, PR China

${ }^{2}$ The Institute of Cardiovascular Sciences and Institute of Systems Biomedicine, School of Basic Medical Sciences, and Key Laboratory of Molecular Cardiovascular Sciences of Ministry of Education, Peking University Health Science Center, Beijing 100191, China

\section{Correspondence to} Dr Yun Yuan, Department of Neurology, Peking University First Hospital, No. 8 Xishiku Street, Xicheng District, Beijing 100034, PR China; yuanyun2002@sohu.com

Received 13 November 2013 Revised 30 July 2014 Accepted 2 August 2014 Published Online First 4 September 2014

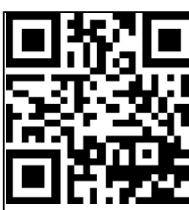

Open Access Scan to access mor free content

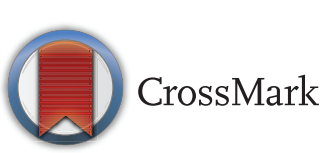

To cite: Xu H, Wang $\mathrm{Z}$, Zheng $\mathrm{L}$, et al. J Clin Pathol 2014:67:999-1005.

\begin{abstract}
Aims Myopathy or neuropathy has been associated with lamivudine/telbivudine therapy in hepatitis B patients. We aim to describe the pathological changes of lamivudine/telbivudine-associated neuromyopathy.

Methods We retrospectively recruited six patients who were diagnosed with nucleotide analogues-associated myopathy or neuropathy. Muscle and nerve biopsy were performed, and the specimens were prepared for the light microscopy and electron microscopy. Genomic DNA was extracted from frozen muscle specimens, and the mitochondrial DNA (mtDNA) content was quantified by real-time PCR
\end{abstract}

Results Recovery of the myopathy can be achieved after the discontinuation or changing the drugs to entecavir. Muscle and nerve biopsy revealed similar changes under either the light or electronic microscopy in all the subjects. Quantitative real-time PCR revealed decrease of mtDNA content in the affected muscle.

Conclusions MtDNA depletion results in mitochondrial dysfunction in the lamivudine/telbivudine-associated neuromyopathy. Myopathy was characterised by mitochondrial dysfunction accompanied with neurogenic damage due to axonal neuropathy. Ultrastructure changes of mitochondria included vacuolisation, simplification of the cristae and homogenised matrix.

\section{INTRODUCTION}

Hepatitis B virus (HBV) infection is a major global health problem, ${ }^{1}$ and a long-term treatment is often required. At present, there are seven approved treatments for patients with chronic HBV infection: two interferon-based therapies (interferon- $\alpha$, pegylated interferon- $\alpha$ ) and five nucleotide analogues (lamivudine, adefovir dipivoxil, entecavir, telbivudine and tenofovir disoproxil fumarate). All nucleotide analogues approved were well tolerated for long-term treatment, but several side effects have also been reported. ${ }^{2} 3$ Lamivudine and telbivudine have been associated with toxic myopathy and peripheral neuropathy. ${ }^{3-5}$ In the worldwide phase III global trial comparing the efficacy and safety of telbivudine versus lamivudine treatment over 2 years, grade 3-4 creatine kinase (CK) elevations (defined as levels $>7$ times the upper limit of normal) were observed in $12.9 \%$ patients receiving telbivudine $(88 / 680)$ and $4.1 \%$ patients receiving lamivudine $(28 / 687) .{ }^{5}$ However, for such nucleotide analogues-associated neuromyopathy, most of the past reports related the clinical symptom and abnormality of laboratory tests, pathological changes have not been described in detail.

The non-selective inhibition of the polymerase $\gamma$ (POLG), the enzyme responsible for mitochondrial DNA (mtDNA) replication, has been implicated as the primary cause of the side effects of nucleotide analogues. ${ }^{2}{ }^{6}$ The mitochondrial toxicity of nucleotide analogues has been demonstrated in vitro. ${ }^{7} 8$ But at the patient level, few reports provided evidence for mitochondrial toxicity, most of which through histochemical stain of the muscle. ${ }^{9}$ The ultrastructure change of the mitochondria has not been described. Though in vitro cell model demonstrated the decrease of mtDNA content during certain nucleotide analogues exposure, ${ }^{10}{ }^{11}$ as for the generally used lamivudine and telbivudine, confirmation of mtDNA depletion underlying the neuromyopathy still requires direct evidence from the patient level.

The muscle or nerve biopsy was very informative for the diagnosis and differential diagnosis of some diseases under the complexity of the clinical practice. In this investigation, we focus our attention on the morphological changes of the affected muscle and nerve during the treatment of lamivudine and telbivudine, either under light or electron microscope (EM). We characterised the common pathological changes of the muscle, nerve and mitochondria during the administration of the two drugs and quantified the mitochondria DNA copy number in the affected muscle. In addition, in our review of the six patients, we found entecavir might not produce mitochondrial toxicity and might be an alternative treatment for the patients susceptible to lamivudine/telbivudine-associated neuromyopathy.

\section{METHODS}

\section{Ethics statement}

This is a retrospectively investigation, and written consents were given by the patients for their information to be stored in the hospital database and used for research. The study protocol was approved by the Institutional Review Board of The Peking University First Hospital. Studies have been performed according to the Declaration of Helsinki.

\section{Patients}

We retrospectively recruited patients diagnosed with nucleotide analogue-associated myopathy at the Peking University First Hospital and analysed their samples from muscle biopsies obtained between 2008 and 2012. All six patients suffered 
chronic HBV infection and developed muscle weakness during long-term lamivudine or telbivudine therapy (table 1). The patients have no comorbidities in present and past history. During the treatment, there is no other co-medication related to neuropathy or myopathy. The clinical data were obtained from the reviews of the patient's medical records.

\section{Pathological study}

Muscle biopsies in six patients and nerve biopsies in three patients were performed after obtaining informed consents. Cryostat sections of the six muscle specimens were stained with the standard histological and enzyme histochemical techniques listed in table 2. For patients 1, 3 and 5, the sural nerve biopsy was performed because of the presence of sensory symptoms simultaneously. The paraffin-embedded specimens were processed for HE, Luxol Fast Blue and Congo red stain. Three muscle specimens (patients 4, 5 and 6) and all three nerve specimens (patients 1, 3 and 5) were prepared for EM analysis. Semithin sections were stained with toluidine blue and viewed under light microscope. Ultrathin sections were stained with uranyl acetate and lead citrate, and were examined using an EM.

\section{Quantification of the mtDNA content in the muscle}

TaqMan probe-based real-time PCR was performed to determine the absolute target quantity in samples. Haemoglobin $\beta$ (HBB) and $\mathrm{NADH}$ dehydrogenase subunit 1 (ND1) gene were used as the amplification targets for the quantification of the genome DNA and mtDNA, respectively. For the HBB gene, the following primers were used: forward: 5'-ACCTCAAGGGCACCTTTGC-3', reverse: 5'-AAAACATCAAGCGTCCCATAGAC-3', and the probe sequence was 5'-TAMRA-CACTGTGACAAGCTGCACGTGGATCC-BH Q2. For the ND1 gene, the following primers were used: forward: 5'-ATTCGATGTTGAAGCCTGAGACT-3', reverse: 5'-TGACCCT TGGCCATAATATGATT- $3^{\prime}$, and the probe sequence was 5'-Hex-TTCGGACTCCCCTTCGGCAAGG-BHQ1. PCR was performed on the Applied Biosystems 7500 Real-Time PCR System with the following procedure: $10 \mathrm{~min}$ at $95^{\circ} \mathrm{C}, 40$ cycles of $15 \mathrm{~s}$ of denaturation at $95^{\circ} \mathrm{C}$ and $60 \mathrm{~s}$ of annealing/extension at $63^{\circ} \mathrm{C}$. PCR products of the $\mathrm{HBB}$ and ND1 gene were inserted into the pBluescript II SK(+) vector(Stratagene), and the purified plasmids were linearised by restriction enzyme HindIII. Serially diluted digested plasmids were used as the standard to generate standard curve. The copies of the sample can be calculated from the standard curve according to their threshold cycle number. The ratio of mtDNA to nuclear DNA (nDNA) was used as a measure of mtDNA content in each specimen.

\section{Statistics}

All data were expressed as mean \pm SD. Statistical analysis was carried out by using SPSS Base V.16.0 software and comparison between groups was assessed by Student $t$ test. Statistical significance was set at $\mathrm{p}<0.01$.

\section{RESULTS}

Clinical symptoms of the patients improved after discontinuation or changing the drug to entecavir

The clinical features are listed in table 1. All patients had a history of HBV infection. Three patients were treated with lamivudine, and the other three with telbivudine. All six patients developed progressive muscle weakness in 1-2 years after initiation of lamivudine or telbivudine therapy. Three patients also complained of muscle pain (patients 1,2 and 6). Three patients were accompanied with numbness in distal end of limbs (patients 1, 3 and 5). The presence of myopathy and neuropathy was also confirmed by the electrophysiological study, high CK levels and muscle and nerve biopsies. Three patients (patients 1, 4 and 6) changed the drug to entecavir $(0.5 \mathrm{mg}$ daily), and Two patients (patients 2 and 3) discontinued nucleotide analogue treatment. After discontinuation or changing the drug to entecavir, all these five patients restored their normal muscle power and the CK levels decreased to the normal level within 3-month follow-up. The muscle pain and limb numbness also disappeared within 3 months. Patient 5 continued lamivudine until now, and no obvious improvement of the weakness was observed.

\section{Neurogenic damage and mitochondrial dysfunction were found in all the affected muscles}

Muscle biopsy findings are summarised in table 2. The prominent and consistent features include variation in fibre size, regeneration, angulated fibres (figure 1A), type II fibre atrophy and numerous cytochrome $c$ oxidase (COX)-deficient fibres (figure 1B). Muscle fibre necrosis was only present in patient 4 . All six patients developed mitochondrial dysfunction to some extent: COX-deficient fibres can be observed in all six patients, the ragged blue fibres $(\mathrm{RBF})$ in five patients (figure $1 \mathrm{C}$ ) and typical and atypical ragged red fibres (RRF) in five patients (figure 1D). NADH-TR enzyme activity deficiency was present in patients 1,2 and 5 . Oil red $\mathrm{O}$ staining demonstrated the increase of lipid content in the fibres in all six patients. Periodic acid Schiff staining revealed glucose deposition in the fibres in two patients.

Table 1 Clinical data of six patients

\begin{tabular}{|c|c|c|c|c|c|c|}
\hline Patients & 1 & 2 & 3 & 4 & 5 & 6 \\
\hline Age, years & 20 & 82 & 26 & 49 & 24 & 25 \\
\hline Sex & Male & Male & Male & Male & Male & Female \\
\hline Drug received by patient & Lamivudine & Lamivudine & Telbivudine & Telbivudine & Lamivudine & Telbivudine \\
\hline Daily dose, mg & 100 & 100 & 600 & 600 & 100 & 600 \\
\hline $\begin{array}{l}\text { Duration of nucleotide analogues therapy before } \\
\text { symptom onset, months }\end{array}$ & 12 & 12 & 12 & 24 & 48 & 12 \\
\hline Interval between symptom onset and evaluation & 6 months & 2 months & 6 months & 12 months & 8 years & 1 month \\
\hline Medication after diagnosis & $\begin{array}{l}\text { Switched to } \\
\text { entecavir }\end{array}$ & Discontinuance & Discontinuance & $\begin{array}{l}\text { Switched to } \\
\text { entecavir }\end{array}$ & $\begin{array}{l}\text { Continued } \\
\text { lamivudine }\end{array}$ & $\begin{array}{l}\text { Switched to } \\
\text { entecavir }\end{array}$ \\
\hline Daily dose, mg & 0.5 & - & - & 0.5 & 100 & 0.5 \\
\hline Recovery time, months & 3 & 3 & 3 & 3 & Not available & 1 \\
\hline
\end{tabular}


Table 2 Pathological features of muscle biopsy

\begin{tabular}{|c|c|c|c|c|c|c|}
\hline Patients & 1 & 2 & 3 & 4 & 5 & 6 \\
\hline Connective tissue proliferation & - & + & - & - & + & - \\
\hline Myonecrosis (H\&E) & - & - & - & + & - & - \\
\hline Regeneration & + & + & + & + & + & + \\
\hline Vacuoles & + & + & - & - & - & - \\
\hline Nuclear internalisation & + & + & - & - & + & + \\
\hline \multicolumn{7}{|l|}{ Muscle fibre } \\
\hline Fibre diameter & Bimodal & Bimodal & Bimodal & Unimodal & Unimodal & Unimodal \\
\hline Hypertrophy fibre & + & + & + & + & + & - \\
\hline small round fibre & - & - & - & - & + & - \\
\hline Angulated fibre & + & + & + & + & + & + \\
\hline Grouped angulated fibre & + & + & + & - & + & - \\
\hline PAS & - & - & - & + & + & - \\
\hline ORO & + & + & + & + & - & + \\
\hline \multicolumn{7}{|l|}{ Mitochondrial change } \\
\hline Raged red fibres (MGT) & + & + & + & + & - & + \\
\hline NADH-TR enzyme activity change & + & + & - & - & + & - \\
\hline COX-negative fibres (COX) & + & + & + & + & + & + \\
\hline Ragged blue fibres (SDH) & + & + & + & + & - & + \\
\hline Type I fibre atrophy (ATPase) & + & - & + & + & + & + \\
\hline Type I fibre hypertrophy (ATPase) & + & - & - & + & + & - \\
\hline Type II fibre atrophy (ATPase) & + & + & + & + & + & + \\
\hline Type II fibre hypertrophy (ATPase) & - & + & - & + & + & - \\
\hline Vessels structure & Normal & Normal & Normal & Normal & Normal & Normal \\
\hline Inflammatory cell infiltration & - & + & - & + & - & - \\
\hline
\end{tabular}

Simplification of the cristae was the prominent change of damaged mitochondria in all the affected muscles subjected to EM

Ultrastructurally, focal damage of the myofibrils can be observed. Abnormality of $\mathrm{Z}$ line including loss, waviness, mild and severe streaming can be observed occasionally. In normal muscle fibres, mitochondria arrange in pairs on both sides of Z-line in longitudinal columns between the myofibrils. However, in longitudinal sections of the specimen of patient 5 , mitochondria decreased obviously and distributed fairly sparsely in some area of some muscle fibres (figure 2A). Mitochondrial proliferation was presented in the RRF fibres. Morphological changes of the mitochondria in the three specimens were basically the same. The shape and size of the mitochondria varied significantly. A quantity of misshapen mitochondria and sparse giant mitochondria can be observed beneath the sarcolemma. The consistent and preponderant changes of the mitochondria were the changes of cristae, including lack of crista definition, simplification of the cristae, parallel cristae that extend most of the way across the profile and homogenised matrix with none crista within the mitochondria, which can be observed frequently in all the muscle specimens subjected to EM (figure 2B). Some mitochondria with circular cristae can be observed (figure $2 \mathrm{~B}$ ). We observed many elongated mitochondria of tubular structures, some of which were branched, with normal diameter between myofibrils in several myofibers (figure 2C, D), which might indicate the increase of the fusion or inefficiency of fission activity of mitochondrial dynamics. ${ }^{12} 13$

\section{Reduction of myelinated fibres with axonal degeneration were found in all the sural nerve biopsies}

Sural biopsies demonstrated moderate reduction of myelinated nerve fibres with axonal degeneration and occasionally regenerated cluster (table 3) (figure 3A). In patient 5, several demyelinated fibres can be observed, but no onion bulb formation. No inflammatory cell infiltration can be observed around vessels or in the epineurium.

\section{Lack of crista definition and homogenised matrix were the main changes of the mitochondria in the nerve biopsies under EM}

Ultrastructural examination confirmed the light microscopy findings above, including axonal degeneration and regeneration (figure 3B). The morphological changes of mitochondria were virtually identical in all the nerve specimens and similar with the features found in the muscle. Most mitochondria were shaped round with varied sizes. The common changes including lack of crista definition and homogenised matrix with none crista within the mitochondria can be observed frequently in the myelinated axons (figure 3C), unmyelinated axons (figure 3D), Schwann's cells and endocapillary cells. Vacuolation of the mitochondria was another common feature and can be observed regularly in all the three nerve biopsies.

\section{MtDNA content reduction was found in all the muscle specimens}

The mean values of the mtDNA content in the six controls and six patients are reported in figure 4 . The mtDNA copy numbers of the six control muscles detected by our quantification system were consistent with the results reported by David Dimmock and his colleagues. ${ }^{14}$ Statistically significant difference was found in the mean value of $\mathrm{mtDNA} / \mathrm{nDNA}$ ratio between the six controls (mean $\pm \mathrm{SD}=1891.76 \pm 815.18$ ) and six patients (mean $\pm \mathrm{SD}=546.45$ $\pm 382.14)(\mathrm{p}<0.01)$. In the six patients, the mtDNA content was reduced to less than $30 \%$ in comparison with the six controls. 


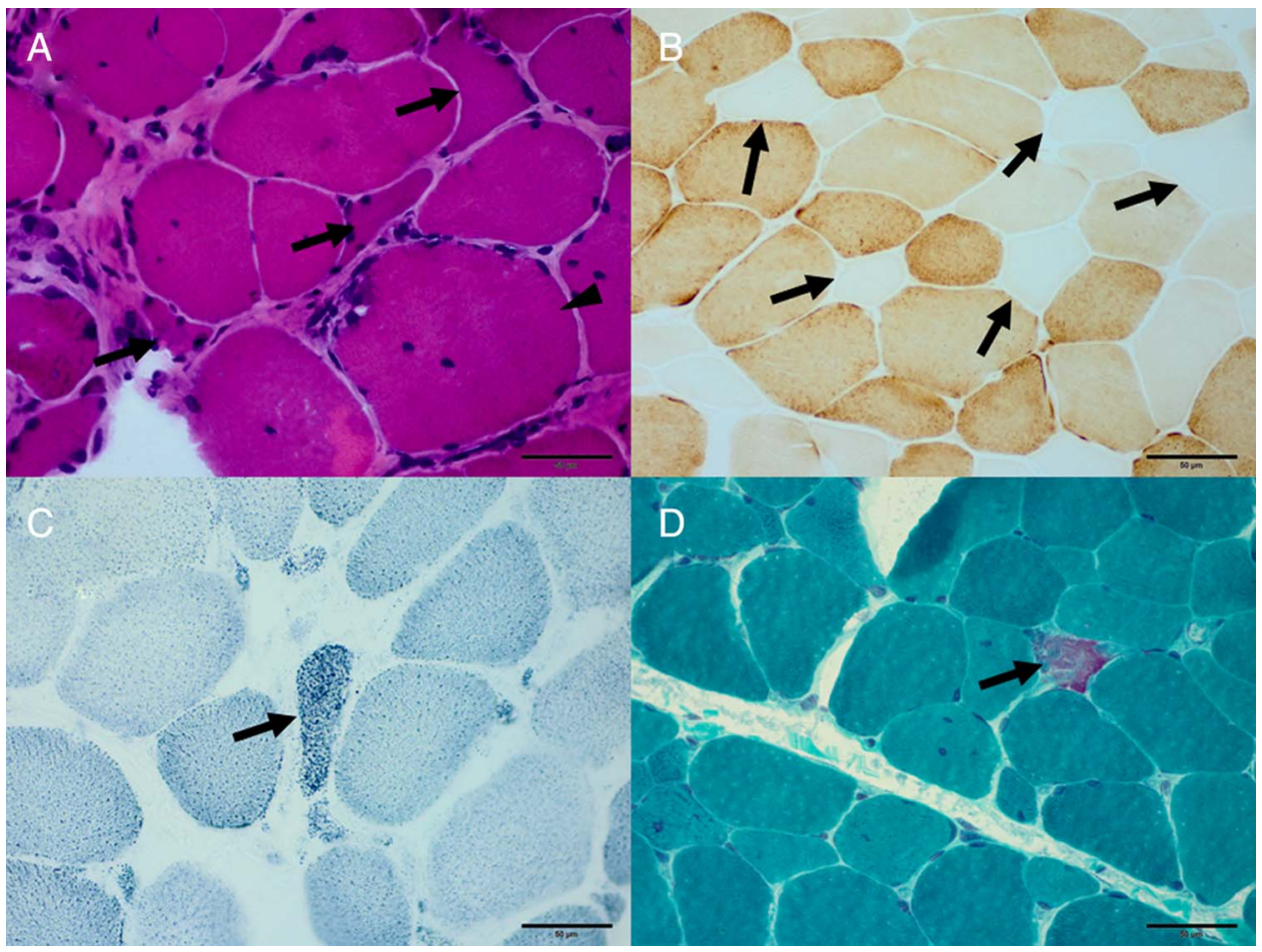

Figure 1 Neurogenic damage and mitochondrial dysfunction in the myopathy during the treatment of lamivudine/telbivudine. (A) Variation of the muscle fibre size, angulated fibres (arrow), hypertrophy fibres (arrowhead) can be observed in patient 5 (haematoxylin and eosin; original magnification, $\times 400$ ). (B) Cytochrome $c$ oxidase-deficient fibres (arrow) in patient 6 (cytochrome $c$ oxidase, original magnification, $\times 400)$. (C) Ragged blue fibres (arrow) in patient 4 (succinate dehydrogenase; original magnification, $\times 400$ ); (D) ragged red fibres (arrow) in patient 6 (modified Gomori trichrome; original magnification, $\times 400)$.

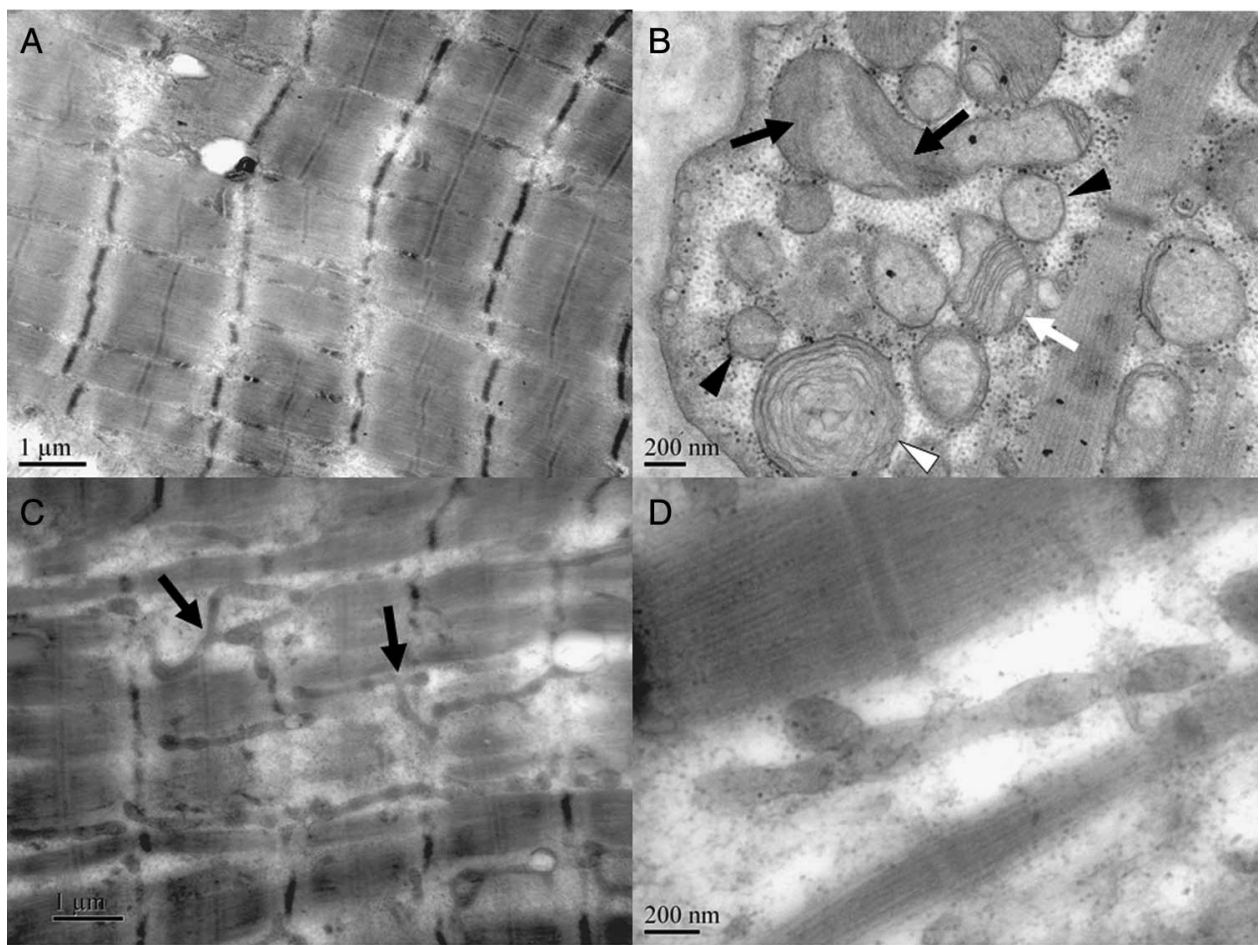

Figure 2 Decreased mitochondria, typical cristae changes and increased fusion activity of mitochondrial dynamics in the myopathy under electronic microscopy. (A) In longitudinal sections of the specimen of patient 5, mitochondria decreased obviously, and distributed fairly sparsely in some areas of some muscle fibres (electron microscope (EM), $20000 \times$ ). (B) Abnormality of the cristae of mitochondria in patient 4, including lack of definition (black arrow), simplification of the cristae (white arrow), parallel cristae (white arrow), homogenised matrix (black arrowhead) and circular cristae (white arrowhead). (C) In patient 5, many elongated mitochondria can be observed in several myofibers, some of which are branched (arrow) $(E M, 20000 \times)$; (D) in patient 5, an elongated mitochondria with normal diameter (EM, $80000 \times)$. 
Table 3 Pathological changes of nerve biopsy

\begin{tabular}{llll}
\hline Patients & $\mathbf{1}$ & $\mathbf{3}$ & $\mathbf{5}$ \\
\hline H\&E and LFB (8 $\mu$ sections) & & & \\
Oedema & - & - & - \\
Vessel constructure & Normal & Normal & Normal \\
Inflammatory cell infiltration & - & - & - \\
Connective tissue proliferation & - & - & - \\
Loss of myelinated axons & Moderate & Moderate & NO \\
Axonal degeneration & + & + & - \\
Demyelination & - & - & + \\
Onionoid structure & - & - & - \\
Congo red stain (8 $\mu$ sections) & - & - & - \\
Toluidine blue Stain (semithin) & & & \\
Vessels constructure & Normal & Normal & Normal \\
Inflammatory cell infiltration & - & - & - \\
Abnormal deposition & - & - & - \\
Connective tissue proliferation & - & - & - \\
Loss of myelinated axons & Moderate & Moderate & NO \\
Axonal degeneration & + & + & - \\
Demyelination & - & - & + \\
Regeneration & + & + & - \\
Onionoid structure & - & - & - \\
Loss of unmyelinated nerve & - & + & - \\
\hline LFB, Luxol Fast Blue. & & &
\end{tabular}

\section{DISCUSSION}

In this study, all the patients have been treated with nucleotide analogues for a long term, in which three with telbivudine and three with lamivudine. The causal link between the development of myopathy and nucleotide analogues administration was clear and convincing. All patients developed their symptoms after the long-term administration of the analogues, and no alternative cause can be found. Muscle weakness and limb numbness improved after the change or discontinuation of the drugs without special management except patient 5 who continued oral lamivudine. Three patients in this retrospective study changed the treatment to entecavir, and muscle strength improved to normal as well. More importantly, with the administration of entecavir, no extra time was needed for these three patients to restore their normal muscle power compared with the two patients who discontinued the nucleotide analogue therapy. This experience suggests that lamivudine/telbivudine-associated neuromyopathy can be cured by withdrawal of the drugs. In addition, entecavir might not produce mitochondrial toxicity and can be an alternative for the lamivudine/telbivudine intolerance, which were supported by another study that showed entecavir displayed no mitochondrial toxicity or DNA POLG inhibition in vitro. ${ }^{15}$

In this study, mitochondrial dysfunction is a common feature in all the patients and the abnormal lipid metabolism might result from the mitochondrial dysfunction. These features were similar with toxic myopathy induced by other nucleotide analogues, $^{9} 16$ but obvious differences were also present. For example, necrosis is a remarkable and consistent feature for the clevudine ${ }^{9}$; the presence of cytoplasm body and T-cell-mediated inflammation was prominent for the zidovudine. ${ }^{16}$ The presence of angulated fibres in all the patients indicated that neurogenic damage was another common feature and the peripheral nerve lesions were confirmed by nerve biopsies. All patients with sensory symptoms presented a subacute axonal neuropathy according to sural biopsy. To our knowledge, other toxic drugs can also damage the muscle or nerve, but mitochondrial abnormalities were seldom produced in the muscle. This will be of great significances in the diagnosis and differential diagnosis of the analogues-associated neuromyopathy in the clinical practice.
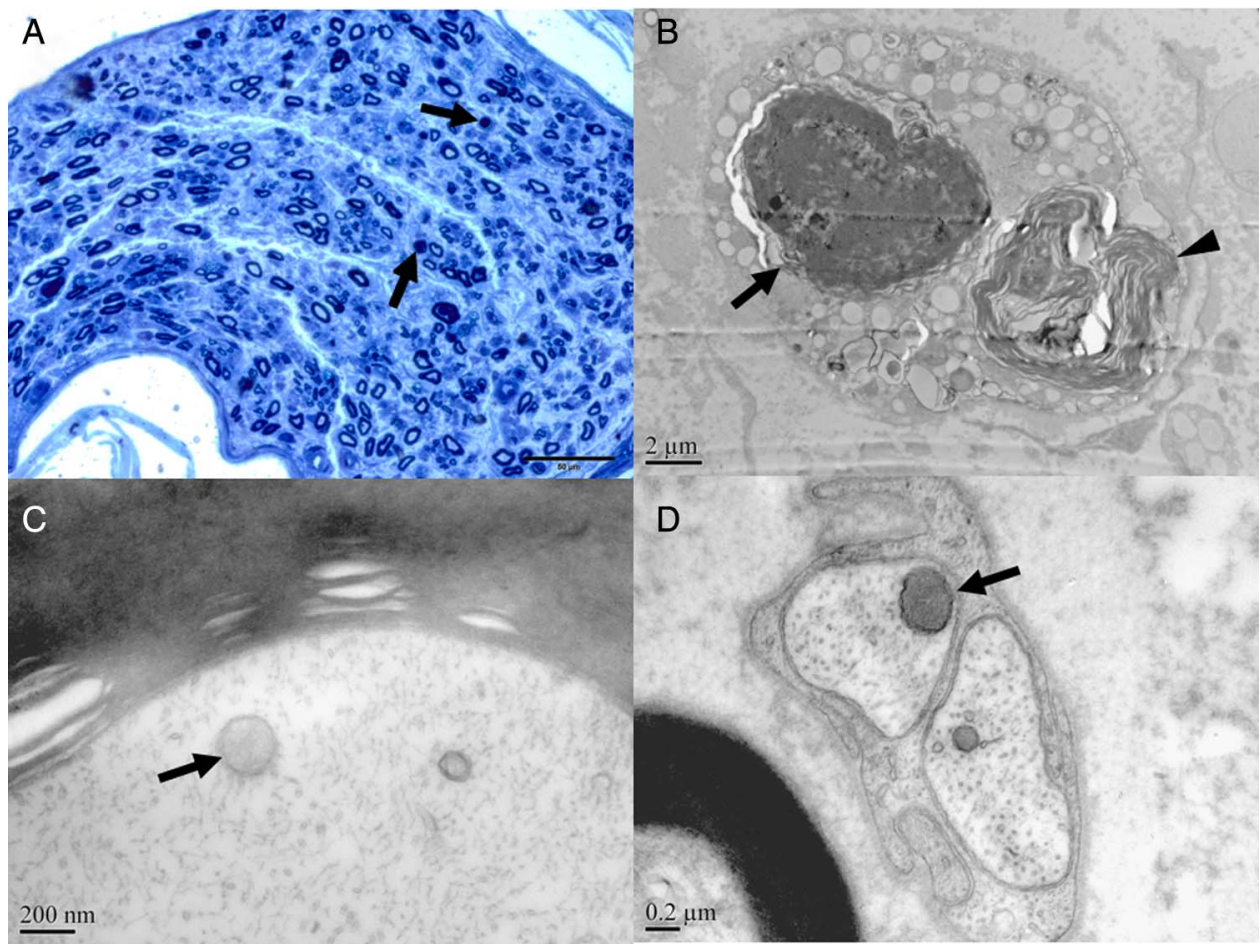

Figure 3 Demyelination, axonal degeneration and homogeneous staining of the mitochondria in neuropathy during the treatment of lamivudine/ telbivudine. (A) Reduction of myelinated nerve fibres, axonal degeneration (arrow) in patient 1. (B) Axonal degeneration (arrow) and Wallerian degeneration (arrowhead) in patient 1 (electron microscope (EM), 8000x). Mitochondria with homogenised matrix (arrow) were observed in the myelinated axons in patient 5 (EM, $80000 x)$ (C), unmyelinated axons (arrow) in patient 1 (EM, $50000 \times)$ (D). 


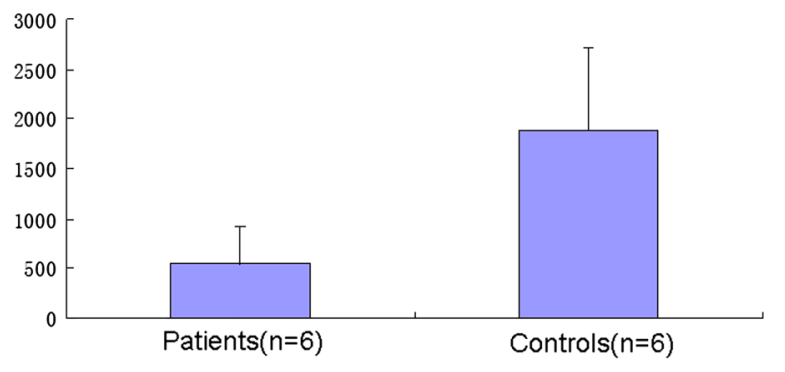

Figure 4 Mitochondrial DNA (mtDNA) content in the affected muscle was reduced to less than $30 \%$ compared with the controls. MtDNA content was measured as mtDNA/nDNA ratio. Bars represent the average $\pm S D$ of values obtained from different samples analysed in triplicate. $n$, number of analysed samples.

In all the six subjects, many mitochondria in the muscle and nerve observed have the common features including lack of crista definition, simplification of the cristae and homogenised matrix. Simplification of the cristae has been related to mtDNA depletion, which leads to the appearance of accumulation of homogeneous material within the mitochondria displacing the normal two-dimensional structures of the mitochondria. ${ }^{11}{ }^{17} \mathrm{In}$ vitro cell model has demonstrated the simplification of the cristae in mtDNA depletion during the exposure of zidovudine, ${ }^{11}$ which is similar to the morphological changes we observed in the patient's tissues. Another in vitro cell model showed circular and curved cristae motifs in mtDNA-depleted $\rho 0$ cells. ${ }^{10}$ We also observed many mitochondria with typical circular cristae, and this change was also related to mtDNA depletion. ${ }^{17}$ The morphological changes of mitochondria were consistent in all six patients, and in all the cell types including muscle fibres, myelinated axons (figure 3C), unmyelinated axons (figure 3D), Schwann's cells and endocapillary cells, which suggest that mitochondrial dysfunction might be a common cause of the nucleotide-associated neuromyopathy. To the best of our knowledge, this is the first report that reveals typical mitochondrial morphological changes during the treatment of lamivudine/telbivudine at the patient level. These morphological changes implicate that mtDNA might decrease during the nucleotide analogue treatment, either in the muscle or in the nerve. The depletion of the mtDNA content was then confirmed in all the muscle specimens. Our data suggest that the mtDNA-depletion-induced mitochondrial dysfunction might be the common cause of the pathological changes observed both in the muscle and the nerve.

The mitochondria were regarded as dynamic organelles that continually fuse and divide. ${ }^{18} 19$ The length, shape, size and number of mitochondria are controlled by the opposing processes of fission and fusion. ${ }^{12}$ Fusion results in fewer and longer mitochondria, whereas fission results in more and shorter mitochondria. ${ }^{13}$ For patient 5, the loss of the mitochondria and elongation of the tubular structure indicate the increasing of the fusion/fission ratio. Fusion is essential for the maintenance of normal mitochondrial function when cells experience metabolic or environmental stresses. $^{20}{ }^{21}$ Fusion rescues stress by allowing functional mitochondria to complement dysfunctional mitochondria by diffusion and sharing of components between organelles. ${ }^{20}$ In the case of patient 5 , the elevation of the fusion/fission ratio might be a compensatory reaction for the mitochondrial dysfunction. Till now, most studies of mitochondrial fusion/fission are limited to the cultured cells, ${ }^{12} 22$ and little is known about the roles that the fusion/fission plays in vivo. Though it has been hypothesised that the mitochondrial fusion might play important roles in the human disease, ${ }^{23-25}$ little evidence has been provided for the involvement of this phenomenon in the pathological process at patient level. Here, we observed the change of mitochondrial dynamics in the muscle biopsy of patient 5 (figure 3C, D). The elevation of fusion/fission ratio was lamivudine induced, and we assume that the fusion here is a protection mechanism for the stress of energy deficiency. The mitochondrial dynamics changes also indicated the mitochondrial dysfunction during the treatment of lamivudine.

In conclusion, mtDNA depletion results in mitochondrial dysfunction in the lamivudine/telbivudine-associated neuromyopathy. Myopathy is characterised by mitochondrial dysfunction accompanied with neurogenic damage due to axonal neuropathy. Mitochondrial changes include simplification of the cristae and homogenised matrix. Mitochondrial fusion was involved in the pathological process.

\section{Take home messages}

- Lamivudine/telbivudine-associated neuromyopathy was characterised by mitochondrial dysfunction accompanied with neurogenic damage due to axonal neuropathy.

- Simplification of the cristae was the prominent change of damaged mitochondria in myopathy.

- Lack of crista definition and homogenised matrix were the main changes of the mitochondria in neuropathy.

- MtDNA depletion might result in mitochondrial dysfunction in the lamivudine/telbivudine-associated neuromyopathy.

Acknowledgements We thank Ms Zhang Qiurong, Ms Zuo Yuehuan, Professor Bu Dingfang and Doctor Liu Hui in Peking University First Hospital for their laboratory assistance.

Contributors $Y Y$ and $H X$ designed research, performed research, analysed data and wrote the paper; ZW, LZ, WZ, HL and SJ performed research and analysed data.

Funding This work was supported by the National Natural Science Foundation of China (no. 30870864) and Twelfth Five Years Plan (no. 2011ZX09307-001-07).

\section{Competing interests None.}

Patient consent Obtained.

Ethics approval Institutional Review Board of The Peking University First Hospital. Provenance and peer review Not commissioned; externally peer reviewed.

Open Access This is an Open Access article distributed in accordance with the Creative Commons Attribution Non Commercial (CC BY-NC 3.0) license, which permits others to distribute, remix, adapt, build upon this work non-commercially, and license their derivative works on different terms, provided the original work is properly cited and the use is non-commercial. See: http://creativecommons.org/ licenses/by-nc/3.0/

\section{REFERENCES}

1 Harkisoen S, Arends JE, van Erpecum KJ, et al. Hepatitis b viral load and risk of hbv-related liver disease: From east to west? Ann Hepatol 2012; 11:164-71

2 Fontana RJ. Side effects of long-term oral antiviral therapy for hepatitis $b$. Hepatology 2009;49:S185-95.

3 Fleischer RD, Lok AS. Myopathy and neuropathy associated with nucleos(t)ide analog therapy for hepatitis b. J Hepatol 2009;51:787-91.

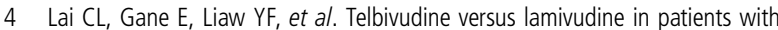
chronic hepatitis b. N Engl J Med 2007;357:2576-88.

5 Liaw YF, Gane $E$, Leung $N$, et al. 2-year globe trial results: telbivudine is superior to lamivudine in patients with chronic hepatitis b. Gastroenterology 2009; 136:486-95.

6 Lee $\mathrm{H}$, Hanes J, Johnson KA. Toxicity of nucleoside analogues used to treat aids and the selectivity of the mitochondrial DNA polymerase. Biochemistry 2003;42:14711-19.

7 Chen $\mathrm{CH}$, Cheng YC. Delayed cytotoxicity and selective loss of mitochondrial DNA in cells treated with the anti-human immunodeficiency virus compound 2',3'-dideoxycytidine. J Biol Chem 1989:264:11934-7. 
8 Vidal F, Domingo JC, Guallar J, et al. In vitro cytotoxicity and mitochondrial toxicity of tenofovir alone and in combination with other antiretrovirals in human renal proximal tubule cells. Antimicrob Agents Chemother 2006;50:3824-32.

9 Seok Jl, Lee DK, Lee $\mathrm{CH}$, et al. Long-term therapy with clevudine for chronic hepatitis $b$ can be associated with myopathy characterized by depletion of mitochondrial DNA. Hepatology 2009;49:2080-6.

10 Gilkerson RW, Margineantu DH, Capaldi RA, et al. Mitochondrial DNA depletion causes morphological changes in the mitochondrial reticulum of cultured human cells. FEBS Lett 2000;474:1-4.

11 Divi RL, Haverkos KJ, Humsi JA, et al. Morphological and molecular course of mitochondrial pathology in cultured human cells exposed long-term to zidovudine. Environ Mol Mutagen 2007;48:179-89.

12 Detmer SA, Chan DC. Functions and dysfunctions of mitochondrial dynamics. Nat Rev Mol Cell Biol 2007:8:870-9.

13 Chan DC. Mitochondrial dynamics in disease. N Engl J Med 2007; 356:1707-9.

14 Dimmock D, Tang LY, Schmitt ES, et al. Quantitative evaluation of the mitochondrial DNA depletion syndrome. Clin Chem 2010;56:1119-27.

15 Mazzucco CE, Hamatake RK, Colonno RJ, et al. Entecavir for treatment of hepatitis $b$ virus displays no in vitro mitochondrial toxicity or DNA polymerase gamma inhibition. Antimicrob Agents Chemother 2008;52:598-605.
16 Dalakas MC, Illa I, Pezeshkpour GH, et al. Mitochondrial myopathy caused by long-term zidovudine therapy. N Engl J Med 1990;322:1098-105.

17 Bourgeois JM, Tarnopolsky MA. Pathology of skeletal muscle in mitochondrial disorders. Mitochondrion 2004;4:441-52.

18 Chan DC. Mitochondrial fusion and fission in mammals. Annu Rev Cell Dev Biol 2006;22:79-99.

19 Okamoto K, Shaw JM. Mitochondrial morphology and dynamics in yeast and multicellular eukaryotes. Annu Rev Genet 2005;39:503-36.

20 Youle RJ, van der Bliek AM. Mitochondrial fission, fusion, and stress. Science 2012:337:1062-5.

21 Schapira AH. Mitochondrial diseases. Lancet 2012;379:1825-34.

22 Suen DF, Norris KL, Youle RJ. Mitochondrial dynamics and apoptosis. Genes Dev 2008;22:1577-90.

23 Nakada K, Sato A, Hayashi J. Mitochondrial functional complementation in mitochondrial DNA-based diseases. Int J Biochem Cell Biol 2009; 41:1907-13.

24 Sato A, Nakada K, Hayashi J. Mitochondrial complementation preventing respiratory dysfunction caused by mutant mtdna. Biofactors 2009;35:130-7.

25 Ono T, Isobe K, Nakada K, et al. Human cells are protected from mitochondrial dysfunction by complementation of DNA products in fused mitochondria. Nat Genet 2001;28:272-5. 